\title{
Effects of Room Temperature and Body Position Change on Cerebral Blood Volume and Center-of-foot Pressure in Healthy Young Adults
}

\author{
Shinichi Demura ${ }^{1)}$, Shunsuke Yamaji ${ }^{2)}$, Tamotsu Kitabashi ${ }^{3)}$, \\ Takayoshi Yamada ${ }^{4)}$ and Masanobu Uchiyama ${ }^{5}$ \\ 1) Department of Physical Education, Kanazawa University \\ 2) University of Fukui Faculty of Medical Science, Morphological and Physiological Sciences, Sports Medicine \\ 3) Yonago National College of Technology \\ 4) Fukui National College of Technology \\ 5) Kanazawa College of Art
}

\begin{abstract}
This study aimed to examine the effects of room temperature and body position changes on cerebral blood volume, blood pressure and center-of-foot pressure (COP). Cerebral oxygenation kinetics and blood pressure were measured by near infrared spectroscopy (NIRS) and volumecompensation, respectively, in 9 males and 9 females after rapid standing from sitting and supine positions in low $\left(12^{\circ} \mathrm{C}\right)$ or normal $\left(22^{\circ} \mathrm{C}\right)$ room temperatures. COP was also measured in a static standing posture for $90 \mathrm{~s}$ after rapid standing. The total hemoglobin $(\mathrm{Hb})$ decreased just after standing. Blood pressure after standing at normal temperature tended to decrease immediately but at low temperature tended to decrease slightly and then to increase greatly. The decreasing ratio of total $\mathrm{Hb}$ and blood pressure upon standing from a supine position at normal room temperatures was the largest of any condition. Total $\mathrm{Hb}$ recovered to a fixed level approximately $25 \mathrm{sec}$ after standing from a sitting position and approximately $35 \mathrm{sec}$ after standing from a supine position. All COP parameters after standing tended to change markedly in the supine position compared to the sitting position, especially at normal temperatures. The COP parameters after standing in any condition were not significantly related to the decreasing ratio of total $\mathrm{Hb}$ but were related to the recovery time of total $\mathrm{Hb}$ after standing. In conclusion, decreasing ratios of total $\mathrm{Hb}$ and blood pressure after standing from a supine position at normal temperatures were large and may affect body sway. J Physiol Anthropol 27(2): 63-70, 2008 http://www.jstage.jst. go.jp/browse/jpa2

[DOI: 10.2214/jpa2.27.63]
\end{abstract}

Keywords: center of foot pressure, near infrared spectroscopy, cerebral symptoms

\section{Introduction}

When healthy people quickly stand up from sitting or supine positions, approximately $500-700 \mathrm{ml}$ of blood moves to the lower body (i.e., lower limbs or abdomen) from the heart, despite the concomitant action of blood pressure regulatory mechanisms (Steinberg, 1980; Andre et al., 1989; Wieling and Shepherd, 1992). Blood pressure is regulated mainly by the baroreceptor reflex, the renin-angiotensin system, and aldosterone release. In particularly, baroreceptors in various organs can detect rapid changes in blood pressure and prevent orthostatic hypotension and fainting. If this blood pressure regulation is not sufficient, systolic pressure temporarily decreases as circulating blood volume diminishes due to pooling of venous blood, and cerebral symptoms such as dizziness and lightheadedness may occur (Mehanoul-Schipper et al., 2001).

Orthostatic hypotension occurs generally when the heart rate increases and the blood pressure decreases (systolic pressure: over $30 \mathrm{mmHg}$, and diastolic pressure: over $15 \mathrm{mmHg}$ ) (Halar and Bell, 1998; Mukai and Lipsitz, 2002). Temporary decreases in oxygen supplied to the cerebrum during orthostatic hypotension may decrease body control while standing.

Continuous cerebral oxygenation monitoring with noninvasive near-infrared spectroscopy (NIRS) can be an indicator of cerebral blood volume changes. Moreover, timeseries recording of the center-of-foot pressure (COP) by a force plate (stabilimeter) allows for accurate and objective body sway monitoring. Variations in cerebral blood volume and blood pressure during posture changes have been examined by many researchers (Harms et al., 2000; Kusano et al., 2000; Kawaguchi et al., 2001) with an emphasis on people with orthostatic symptoms and the elderly. However, few have examined the relationship between cerebral blood volume and 
body sway observed immediately after postural changes. Moreover, even in healthy young people, physiological variations, such as cerebral blood volume, blood pressure and autonomic nervous function, occur temporarily during postural changes (Mehagnoul-Schipper et al., 2000; Kawaguchi et al., 2001). Tanaka et al. (1994) observed changes in orthostatic hypotension in Japanese and Swedish people and reported that the diastolic pressure in the supine position and the systolic pressure while standing were significantly lower in Japanese than in Swedish people. In addition, they inferred that the Japanese have less body stability during standing as a result of orthostatic hypotension.

We hypothesized that standing from a supine or sitting position produces a temporary reduction in cerebral perfusion and a subsequent increase in body sway even in healthy young people. Furthermore, if the temporary reduction in cerebral perfusion after postural changes increases body sway, it is assumed that a significant postural change will further exacerbate body sway. Moreover, orthostatic dizziness is mainly attributed to decreased blood pressure from a temporary decrease in venous return. Blood pressure regulation increases the peripheral vascular resistance with an increase in heart rate leading to stabilization of blood pressure. Therefore, we hypothesized that the decrease of cerebral perfusion is restrained in low ambient temperatures because vasoconstriction caused by cold temperatures will lead to lower reductions of blood pressure.

This study aimed to compare variations in cerebral oxygenation, blood pressure, and COP after standing from the sitting and supine positions at normal and low room temperatures for healthy young Japanese adults.

\section{Methods}

\section{Subjects}

The subjects were 18 healthy young adults ( 9 males, M age $=20.6$ years, $\mathrm{SD}=1.4, \mathrm{M}$ height $=176.1 \mathrm{~cm}, \mathrm{SD}=8.1, \mathrm{M}$ weight $=70.3 \mathrm{~kg}, \mathrm{SD}=9.3 \mathrm{~kg}, 9$ females, $\mathrm{M}$ age=20.2years, $\mathrm{SD}=1.2, \mathrm{M}$ height $=164.6 \mathrm{~cm}, \mathrm{SD}=3.5, \mathrm{M}$ weight $=56.7 \mathrm{~kg}$, $\mathrm{SD}=4.5)$. Subjects did not habitually use any systemic medicines. Written informed consent was obtained from all subjects and their parents after a full explanation of the experimental purpose and protocol. This experimental protocol was approved by the ethics committee at Kanazawa University.

\section{Materials}

\section{1) Stabilimeter}

An Anima's stabilimeter (G5500, Japan) was used for the measurement of the COP. This instrument calculates the COP of vertical loads from three vertical load sensors placed at the peak of an isosceles triangle on a level surface. Data were recorded at a sampling frequency of $20 \mathrm{~Hz}$. Eleven COP parameters with high trial-to-trial and day-to-day reliabilities (Demura et al., 2001; Yamaji et al., 2001) were selected from the following 4 domains: distance (mean path length, root mean square), velocity (mean velocity of X- and Y-axis, root mean square of sway velocity), area (area surrounding mean path length, area surrounding maximal amplitude rectangle for each axis), and amplitude distribution (standard deviation of $\mathrm{X}$ - and Y-axis, standard deviation of X- and Y-axis velocity).

\section{2) Cerebral tissue oxygenation monitor}

Near-infrared spectroscopic (NIRS) instruments (NIRO-300, Hamamatsu Photonics, Japan) measured cerebral tissue oxygenation (total hemoglobin (Total $\mathrm{Hb}$ ), the tissue oxygen saturation index (TOI), the oxygenated hemoglobin (Oxy-Hb), and the deoxygenated hemoglobin (Deoxy-Hb) during posture changes. Changes in total $\mathrm{Hb}$ volume indicate changes in cerebral blood volume. The probe of the NIRO-300 contains a light source filtered at 775, 813,850, and $913 \mathrm{~nm}$ and three optical detectors placed $5 \mathrm{~cm}$ from the light source. The changing volumes of oxy-/deoxy $\mathrm{Hb}$ and oxidized/reducedcytochrome aa3 are determined and analyzed with an algorithm incorporating the modified Beer-Lambert law (Hamaoka et al., 1996, 2003). The slope of light attenuation versus distance is determined by estimating the relative absorption coefficients of tissue, i.e., the non-scattered light. Scattered light is delivered via two fiber-optic light detectors to a photomultiplier at $0.5 \mathrm{sec}$ intervals. It is hypothesized that the oxygenation kinetics determined by the NIRO-300 are measured at a depth of about $25 \mathrm{~mm}$ from the skin, because the mean path length is half the distance between the light source and the detector. The total absorbance of light incident by the tissue was the sum of the absorbance by Oxy-Hb and Deoxy$\mathrm{Hb}$ in the blood and other tissues.

\section{3) Blood pressure}

Arterial pressure in the finger was measured with the volume-compensation method (Radia press RBP-100, KANDS, Japan). Averages of systolic and diastolic blood pressure values measured by noninvasive continuous blood pressure monitoring devices were calculated every second. The measurement principle of this instrument is almost the same as the commonly used Portapres (Finapres Medical System) although the algorithms of pressure correction are different. This instrument determines absolute values of arterial blood pressure from light volume variation corrected by calibration, the ratio of pressure pulse wave (standard values) to photoelectric volume pulse wave. After measuring blood pressure at the upper arm during rest by the oscillometric method, this device detects variations of blood hemoglobin with blood flow volume by optical wavelength irradiation $(640 \mathrm{~nm})$ to the skin from an emission sensor attached to the ear lobule while providing light pressure (about $100 \mathrm{mmHg}$ ). Because the variations of blood hemoglobin are relative, the blood pressure is corrected with the ratio of the value during rest as measured by the oscillometric method. The photoelectric volume pulse wave data is corrected with this value. Absolute blood pressure values were determined with a prepared algorithm for blood pressure computation. Emission sensors consist of a photoelectronic sensor and a body motion sensor. The former detects reflected light volume variation in 
the blood vessel from optical wavelength irradiation $(640 \mathrm{~nm})$, and the latter detects reflected light volume variation on a surface by the photoelectronic sensor. It is designed to eliminate measurement errors that result from changes in the photoelectric volume pulse wave by body motion.

Nakada et al. (2007) reported a high trial-to-trial reliability of blood pressure measured by this instrument and close agreement with the Portapres during exercise.

\section{Experimental procedure}

The experimental design was a cross-over design where the subjects participated in all of the experimental conditions (room temperatures: normal $\left(22^{\circ} \mathrm{C}\right)$ and low $\left(12^{\circ} \mathrm{C}\right)$; body position during rest: sitting and supine). Each subject was measured on the same day with an interval of 30 min between trials, and the trials were randomized in terms of the order of the conditions. The humidity was kept at 30\% for all conditions. The NIRS probe was attached to the subject's frontal plane, and cerebral oxygenation was measured for 1 min while sitting in a normal temperature room. The NIRS probe remained attached until the end of all experimental conditions. In all conditions, the mean value of cerebral oxygenation while sitting in a normal temperature room was set as the criteria value $(100 \%)$. The changes in continuous blood pressure were measured at the ear lobule with the Radia press RBP-100. During standing with the measurement of COP, cerebral oxygenation was continually measured. The subjects entered the experimental room which was maintained at a low or normal temperature, and remained quiet in either a sitting or a supine position for $10 \mathrm{~min}$ in order to adapt to the room environment. Then, they stood up and the COP was measured in an upright posture for $90 \mathrm{sec}$. They were instructed to respire naturally and to stabilize their body posture quickly. Moreover, to reduce the influence of muscle contractions, they were instructed not to overstrain their muscles. Following this procedure, each experimental condition (room temperature and body position during resting conditions) was repeated three times. The switch was set to the lateral region of the thigh in order to respond to standing. The sensor was switched when the subject stood vertically on the floor, and the COP was measured. The event signal was entered into the NIRS recorder at the same time.

\section{Data analysis}

The cerebral oxygenation by NIRS in each condition was compared to the mean value at a sitting position in the normal temperature room. The trial-to-trial reliabilities of the COP and the changing ratio of the cerebral oxygenation before and after standing were examined using an intra-class correlation coefficient (ICC). The reliability of the time-series change of cerebral oxygenation and blood pressure were examined by the cross correlation coefficient. The mean differences in COP after standing and the changing ratio of cerebral oxygenation before and after standing between conditions (room temperature and body position during rest) were examined using the two-way ANOVA. For the multivariate comparison, Tukey's HSD test was used. The recovery time from the temporary decrease of total $\mathrm{Hb}$ after standing was calculated in each condition, and the relationship between this time and the COP parameters was examined using Pearson's correlation coefficient. A probability level of 0.05 was indicative of statistical significance.

\section{Results}

The ICC of the COP between trials was over 0.75 (ICC $=$ 0.76-0.83) in all conditions. Moreover, the cross-correlation coefficient of the time-series change of cerebral oxygenation and blood pressure was over $0.8\left(\mathrm{r}_{\mathrm{xy}}=0.80-0.94\right)$. Therefore, the data of the third trial, exposure for a long time, was used in the subsequent analysis.

Figure 1 shows the time series change in the parameters regarding total $\mathrm{Hb}$ in the frontal head plane, a cerebral blood volume indicator, and blood pressure in each body position and room temperature. Total $\mathrm{Hb}$ time-series values were calculated relative to the value at rest while sitting at $22^{\circ} \mathrm{C}$. The total $\mathrm{Hb}$ just after standing decreased significantly more from the supine position under normal room temperature conditions compared with the other conditions. Average blood pressure after standing at normal temperature tended to decrease immediately but that at low temperature tended to decrease slightly and then increase greatly.

Figure 2 shows the result of the two-way ANOVA (room temperature $\times$ body position during rest) for a changing ratio of cerebral oxygenation kinetics before and after standing. There were no significant interaction factors for any of the parameters, and the changing ratio for the supine position in normal temperature conditions was the largest of any of the conditions. The decreasing ratio of these parameters at sitting and supine positions in low-temperature conditions tended to be smaller than that in normal temperature conditions.

Table 1 shows the results of the two-way ANOVA (room temperature $\times$ body position during rest) for the COP parameters after standing. There were significant differences in the main factors (body position during rest) in all of the parameters, except for the standard deviation of the Y-axis. The body sways for supine positions at normal and low temperatures were significantly larger than those for the sitting position.

In both temperature rooms, the recovery of total $\mathrm{Hb}$ after standing from a sitting position took about $25 \mathrm{sec}$, but that from a supine position took about $35 \mathrm{sec}$. The two-way ANOVA of the recovery time (room temperature $\times$ body position during rest) revealed significant differences in the main factors (body position during rest); the recovery times at a supine position were significantly longer than those at a sitting position. Table 2 shows the correlations between COP parameters and decreasing ratio and recovery time of total $\mathrm{Hb}$ upon standing. The COP parameters after standing in any condition were not significantly related to the decreasing ratio 

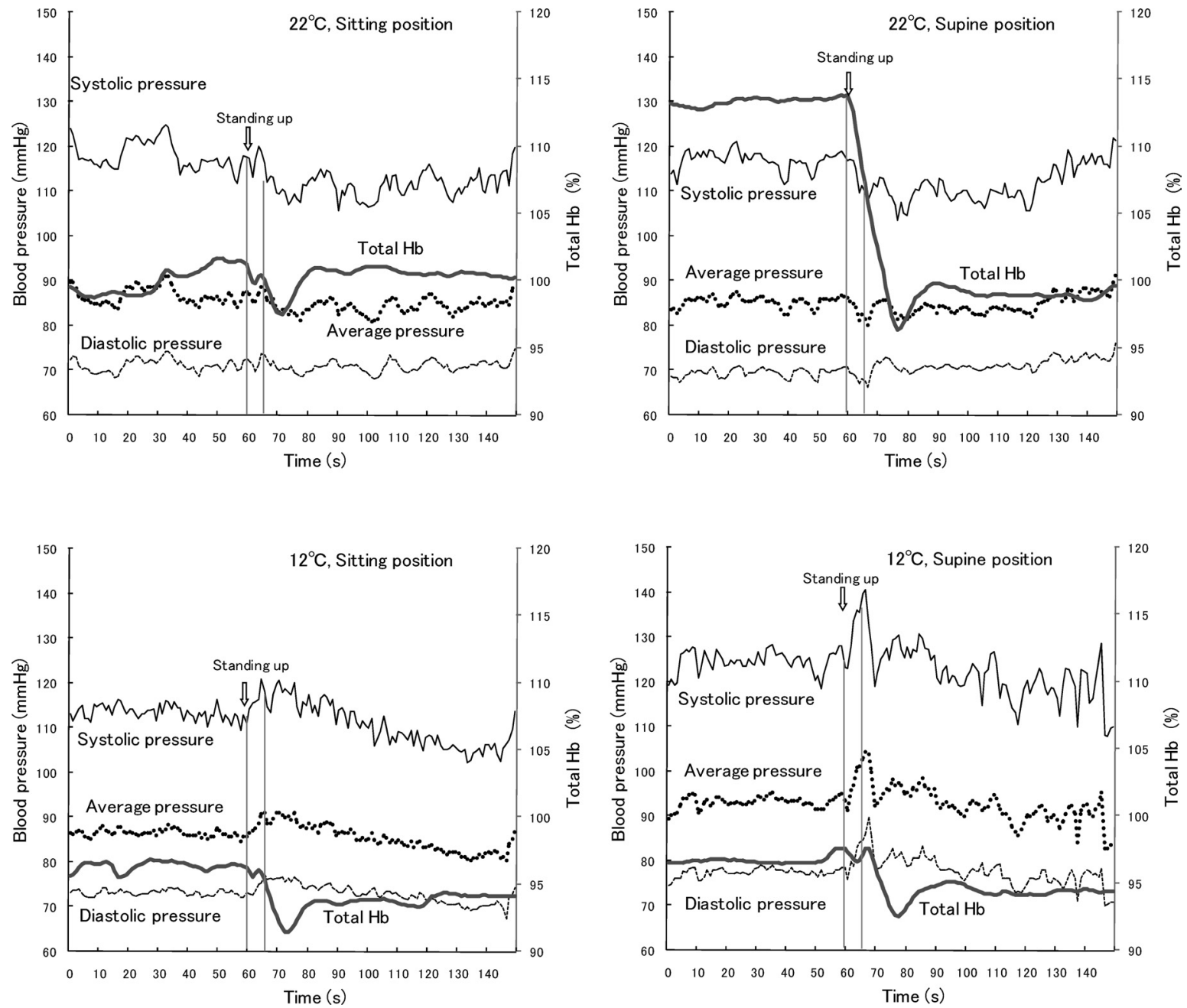

Fig. 1 Changes in total hemoglobin and blood pressure in each body position and room temperature. Values are expressed as the mean for each sampling time. Total $\mathrm{Hb}$ values were calculated relative to the value at rest while sitting at $22^{\circ} \mathrm{C}$.

of total $\mathrm{Hb}$ but showed significant correlations with the recovery time of total $\mathrm{Hb}$ after standing (e.g., root mean square or standard deviation of Y-axis; $\mathrm{r}=0.47-0.76, p<0.05$ ).

\section{Discussion}

Healthy people do not experience large changes of body posture or syncope because of blood pressure regulation (Steinberg, 1980). However, they often feel symptoms such as dizziness or lightheadedness caused by the temporary decrease of blood pressure (Mehanoul-Schipper et al., 2001). Orthostatic hypotension resulting from impaired blood pressure regulation causes a temporarily-diminished oxygen supply to the brain (Kawaguchi et al., 2001). We hypothesized that when the outside air temperature is low, orthostatic hypotension is controlled by vasopressor adaptation. Moreover, since the movement of circulating blood to the lower body after standing from a supine position is larger than that from a sitting position, this symptom may become more marked.

\section{1) Cerebral oxygenation kinetics after standing}

The time-series changes of blood pressure in this study may support this hypothesis which the changing volume corresponds to a significant posture change because the blood flow volume from the head to the lower limbs increases as a result of gravity when standing up from a supine position rather than sitting position. Therefore, if the decline in blood supply causes cerebral symptoms, this symptom may occur more readily when standing up from a supine position than from a sitting position. Few have studied the influence on body sway of the temporary decline in the cerebral blood volume after standing. The findings of previous studies regarding the physiological response, such as the cerebral oxygenation available after changing posture, may be summarized as follows: after standing, the blood pressure and heart rate increase, and the oxy- $\mathrm{Hb}$ and total $\mathrm{Hb}$ decrease, whereas deoxy-Hb increases (Colier et al., 1997; Mehagnoul-Schipper, 2000; Kawaguchi et al., 2001; Tanaka et al., 2003). Moreover, since Total- $\mathrm{Hb}$ and $\mathrm{Oxy}-\mathrm{Hb}$ decrease after standing, it is inferred that the cerebral blood volume decreases during the 


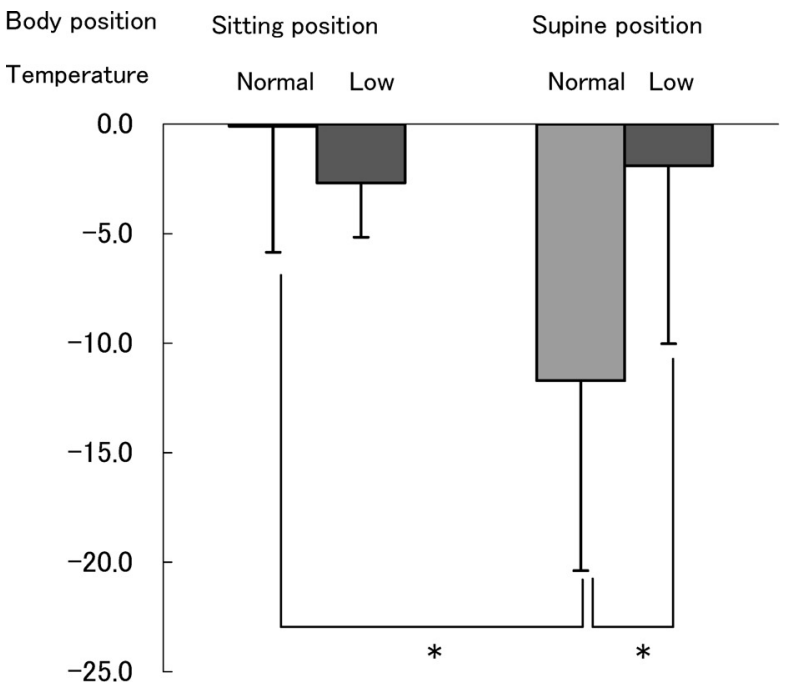

$(\%)$

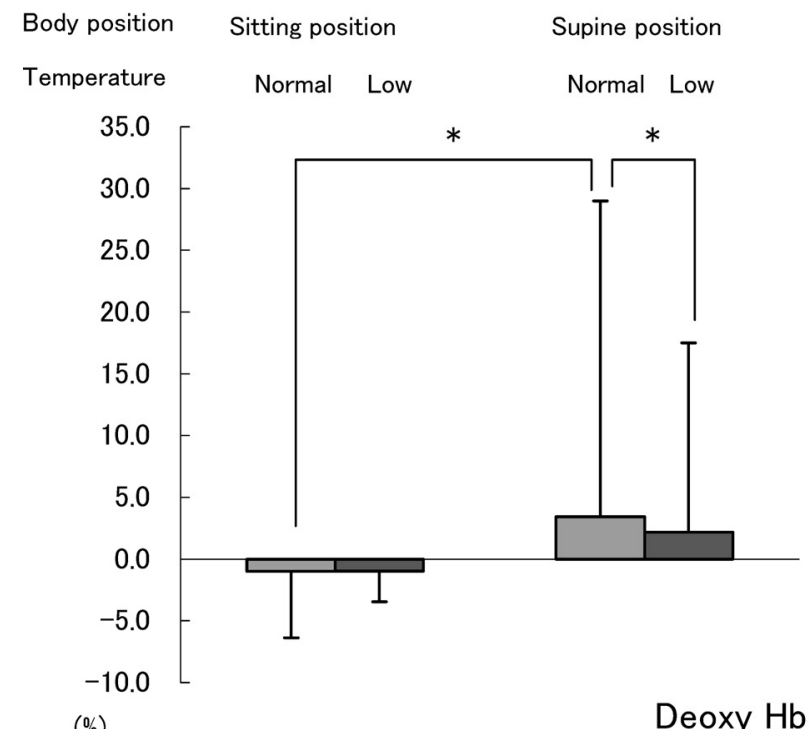

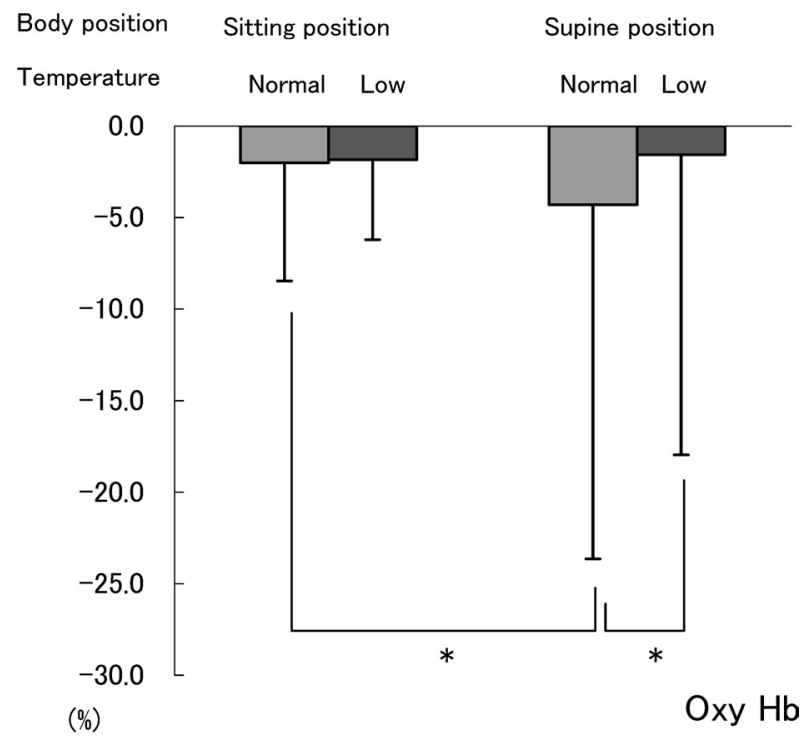

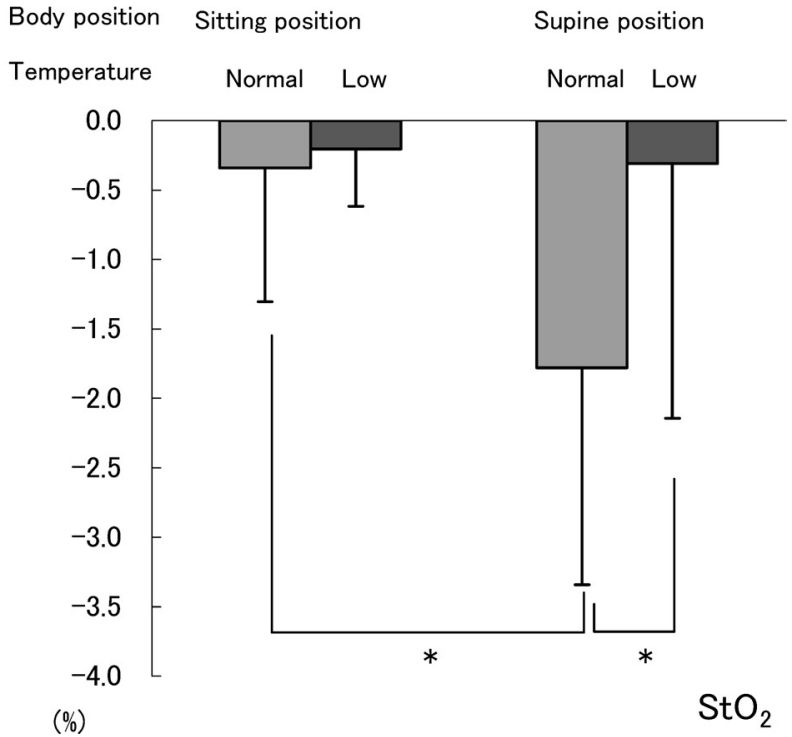

Fig. 2 The changing ratio of cerebral hemodynamics before and after standing. Two way ANOVA (Temperature $\times$ Body position during rest) and post-hoc (Tukey's HSD) reveal the differences in the changing ratio. *: $p<0.05$.

rapid posture change.

The results of this study regarding the changing tendency of Oxy-Hb, total Hb, TOI and Deoxy-Hb agreed with those of previous studies (Colier et al., 1997; Madsen et al., 1998; Kusano et al., 2000; Kawaguchi et al., 2001; Tanaka et al., 2002). The circumvascular vessels change in response to the room temperature and become smaller under low-temperature conditions. The circulating blood volume is lower because the circumvascular vessels are smaller. Moreover, the blood supply to the cerebrum with the decline in blood pressure may be small under low temperatures, because the change of cerebral oxygenation parameters after standing was smaller than that under normal temperatures. In other words, the temperature of the room may exert a greater influence on cerebral oxygenation compared to the body position change (sitting to standing or supine to standing).

\section{2) COP changes after standing}

Body sway after standing may be influenced by the resting position, that is, standing from a supine position rather than from a sitting position, regardless of the outside air temperature. As stated above, although the decrease of total $\mathrm{Hb}$ at low temperatures was small compared with that at normal temperatures, there were no significant differences in COP parameters between both room temperatures. Therefore, in healthy people, the temporary decrease in the volume of cerebral blood after standing, or the temporary decrease in blood pressure, may not be the main body sway factor that interrupts body posture. 


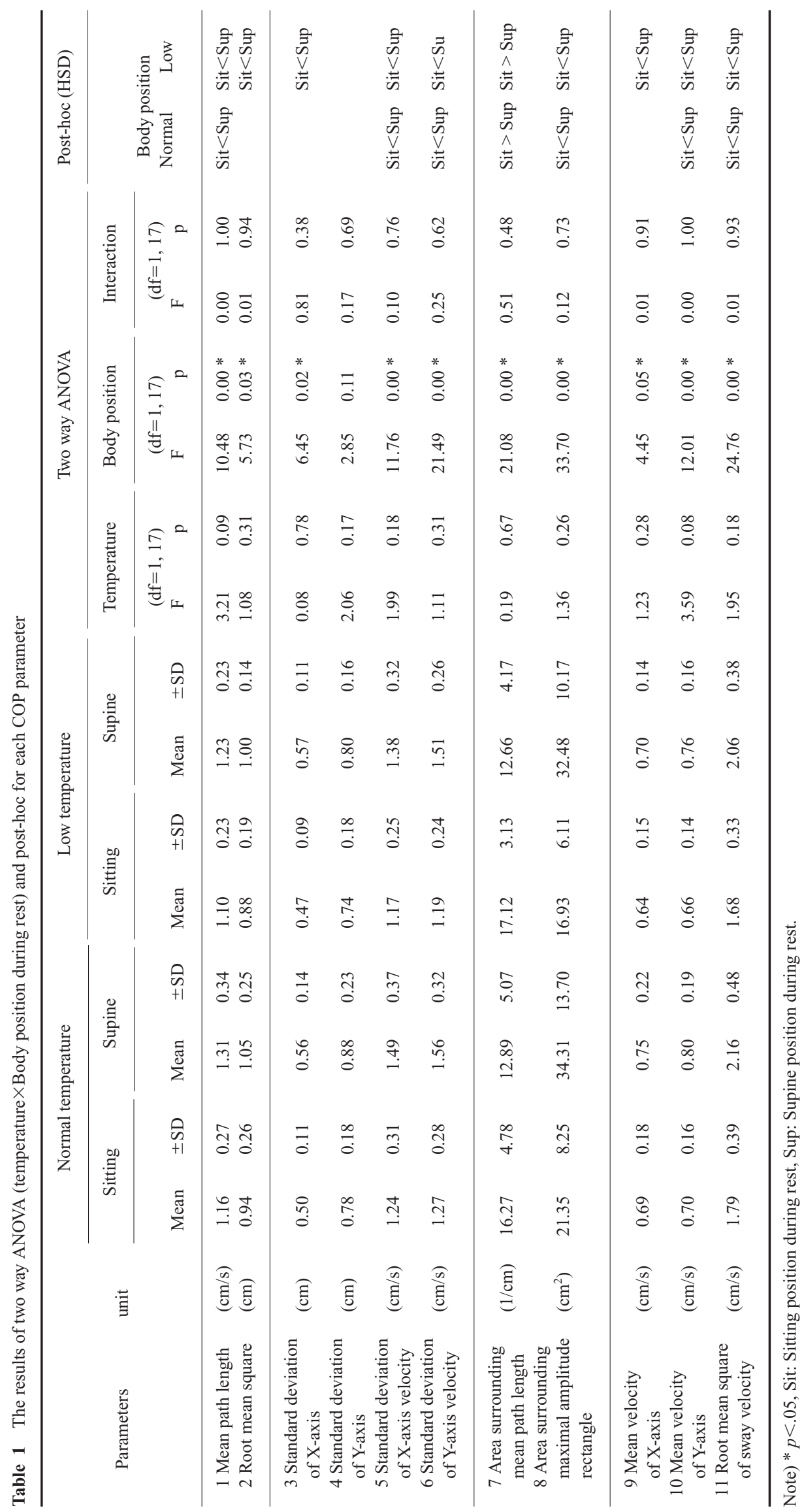


3) The relationships among cerebral oxygenation, blood pressure changes, and body sway after standing

It has been reported that blood pressure regulation in healthy people leads to blood pressure recovery approximately $20-30$ sec after rapid standing (van Lieshout et al., 2001). This study supports these findings. Moreover, the total $\mathrm{Hb}$ in the cerebrum recovered approximately $20-30 \mathrm{sec}$ after a temporary decrease with standing. This recovery corresponded to blood pressure recovery. There is a significant difference between recovery times in resting positions (i.e., sitting and supine positions), and was significantly longer in the supine position. Moreover, the total $\mathrm{Hb}$ markedly decreased after standing, and did not recover to the resting level after $1 \mathrm{~min}$, except in the case of sitting at normal temperature conditions (Fig. 1). Tanaka et al. (2003) reported that patients with orthostatic intolerance did not recover their total $\mathrm{Hb}$ and oxy-Hb of cerebral blood after standing. Further, they suggested that the delay in the recovery of the cerebral oxygenation kinetics is closely related to chronic fatigue. The present results also showed the relationship between the recovery time of total $\mathrm{Hb}$ in the cerebrum and body sway parameters. Therefore, it is possible that the delay of blood pressure regulation influences body sway and largely depends on differences of the resting position (sitting and supine positions) rather than the outside air temperature.

\section{4) Conclusion}

In conclusion, cerebral oxygenation decreases temporarily just after standing in any condition, but may be affected by room temperature more than body position change. Body sway is related to the recovery time of total $\mathrm{Hb}$ after a temporary decrease with standing.

\section{References}

Andre JL, Petit JC, Gueguen R, Spyckerelle Y, Deschamps JP (1989) Blood pressure variations from clinostatism to orthostatism. Arch Mal Coeur Vaiss 82: 1027-1032

Colier WN, Binkhorst RA, Hopman MT, Oeseburg B (1997) Cerebral and circulatory haemodynamics before vasovagal syncope induced by orthostatic stress. Clin Physiol 17: 83-94

Demura S, Yamaji S, Noda M, Kitabayashi T, Nagasawa Y (2001) Examination of parameters evaluating the center of foot pressure in static standing posture from the viewpoints of trial-to-trial reliability and interrelationships among parameters. Equilibrium Res 60: 44-55

Hamaoka T, Iwane H, Shimomitsu T, Katsumura T, Murase N, Nishio S, Osada T, Kurosawa Y, Chance B (1996) Noninvasive measures of oxidative metabolism on working human muscles by near-infrared spectroscopy. J Appl Physiol 81: 1410-1417

Hamaoka T, Katsumura T, Murase N, Sako T, Higuchi H, Murakami M, Esaki K, Kime R, Homma T, Sugeta A, Kurosawa Y, Shimomitsu T, Chance B (2003) Muscle oxygen consumption at onset of exercise by near infrared 
spectroscopy in humans. Adv Exp Med Biol 530: 475-483

Halar EM, Bell KR (1998) Rehabilitation Medicine Principles and Practice. In DeLisa JA ed. Contracture and other deleterious effects of immobility. Lippincott-Raven, Hagerstown, 23-43

Harms MP, Colier WN, Wieling W, Lenders JW, Secher NH, van Lieshout JJ (2000) Orthostatic tolerance, cerebral oxygenation, and blood velocity in humans with sympathetic failure. Stroke 31: 1608-1614

Kawaguchi T, Uyama O, Konishi M, Nishiyama T, Iida T (2001) Orthostatic hypotension in elderly persons during passive standing: a comparison with young persons. J Gerontol A Biol Sci Med Sci 56: M273-280

Kusano E, Yorifuji S, Okuno M, Nakanishi F, Imaoka H, Matsuno Y, Abe K, Hayakawa K (2000) Skin hemodynamics during change from supine to lateral position. J Neurosci Nurs 32: 164-168

Madsen P, Pott F, Olsen SB, Nielsen HB, Burcev I, Secher NH (1998) Near-infrared spectrophotometry determined brain oxygenation during fainting. Acta Physiol Scand 162: 501-507

Mehagnoul-Schipper DJ, Vloet LC, Colier WN, Hoefnagels WH, Jansen RW (2000) Cerebral oxygenation declines in healthy elderly subjects in response to assuming the upright position. Stroke 31: 1615-1620

Mehagnoul-Schipper DJ, Colier WN, Jansen RW (2001) Reproducibility of orthostatic changes in cerebral oxygenation in healthy subjects aged 70 years or older. Clin Physiol 21: $77-84$

Mukai S, Lipsitz LA (2002) Orthostatic hypotension. Clin Geriatr Med 18: 253-268

Nakada M, Demura S, Yamaji S, Kondou S (2007) Comparison of blood pressure changes during intermittent progressive workload exercise with a bicycle ergometer measured by two different noninvasive continuous blood pressure monitoring devices. J Edu Health Sci (in press)

Steinberg FU (1980) The immobilized patient-functional pathology and management. Plenum Medical Book, New York, 121-134

Tanaka H, Thulesius O, Borres M, Yamaguchi H, Mino M (1994) Blood pressure responses in Japanese and Swedish children in the supine and standing position. Eur Heart J 15: 1011-1019

Tanaka H, Matsushima R, Tamai H, Kajimoto Y (2003) Impaired postural cerebral hemodynamics in young patients with chronic fatigue with and without orthostatic intolerance. J Pediatr 140: 412-417

van Lieshout JJ, Pott F, Madsen PL, van Goudoever J, Secher NH (2001) Muscle tensing during standing: effects on cerebral tissue oxygenation and cerebral artery blood velocity. Stroke 32: 1546-1551

Wieling W, Shepherd JT (1992) Initial and delayed circulatory responses to orthostatic stress in normal humans and in subjects with orthostatic intolerance. Int Angiol 11:69-82

Yamaji S, Demura S, Noda M, Nagasawa Y, Nakada M, Kitabayashi T (2001) The day-to-day reliability of parameters evaluating the body center of pressure in static standing posture. Equilibrium Res 60: 217-226

Received: August 9, 2007

Accepted: December 27, 2007

Correspondence to: Shunsuke Yamaji, University of Fukui Faculty of Medical Science, Morphological and Physiological Sciences, Sports Medicine, 23-3, Shimoaizuki, Matsuoka-cho, Yoshida-gun, Fukui 910-1193, Japan

Phone: +81-776-61-3111

Fax: +81-776-61-8145

e-mail: yamaji@u-fukui.ac.jp 